\title{
PERFORMANCE ANALYSIS OF TIME-FREQUENCY TECHNIQUE FOR THE DETECTION OF SMALL SHIPS IN SAR IMAGERY AT LARGE GRAZING ANGLE AND MODERATE METOCEAN CONDITIONS
}

\author{
Domenico Velotto ${ }^{l}$ and Björn Tings ${ }^{1}$ \\ ${ }^{1}$ DLR, German Aerospace Center, Remote Sensing Technology Institute \\ 28199 Bremen, Germany
}

\begin{abstract}
This paper addresses the performance of timefrequency based techniques for the detection of small ships (length less than 30m) under side-looking synthetic aperture radar (SAR) limiting conditions. The aim of this work is to assess this technique for improving TerraSAR-X (TS-X) near real time (NRT) ship detection service. The goals are achieved by processing both the co-polarized single look complex channels (HH and VV) of TS-X data where ships have been identified by their self-reporting messaging system. The results show that the target-to-clutter ratio does not improve significantly for the detection of small ships under the conditions investigated.
\end{abstract}

Index Terms - SAR, ship detection, timefrequency methods, performance analysis

\section{INTRODUCTION}

The surveillance of small ships in open ocean is an important aspect in the maritime domain awareness because of the large number of unlawful activities, such as illegal fishing, human trafficking, smuggling etc., involving this means of transport. It is clear that a non-cooperative monitoring system is essential in this case. Satellite synthetic aperture radar (SAR) ship detection is a well-established non-cooperative monitoring procedure of the maritime environment. The detection of ships with length up to one order of magnitude the spatial resolution of the SAR imagery is however challenging and the success depends on a large number of factors, e.g. construction material of the ship, metocean conditions, just to name the most obvious.

An open-ended question in SAR ship detection is the minimum detectable ship size, which is often simplified with the minimum ship length. There are three different approaches that have been used in the literature: 1) in [1]-[3] a semi-empirical prediction mode has been developed merging an ocean radar backscatter geophysical model function (GMF) model and fitting a function between the physical parameter ship length and the measured maximum radar cross section (RCS) of several ship samples; 2) in [4] a binary classifier trained using detected and nondetected ships samples in real TerraSAR-X (TS-X) SAR data, has been used to develop a data-driven ship detectability model; 3) in [5] a direct observation method has been employed by visual interpretation of very high resolution (VHR) SAR imagery over predefined test sites where small boats were deployed. All the three approaches agree that using the radar backscatter information the minimum ship length strongly decreases either with increasing wind speed and decreasing incidence angle. To show these effects, Figure 1 provides the probability of detection maps obtained as outcome of the data-driven detectability model developed in [4] using over $150 \mathrm{X}$-band high resolution StripMap TS-X HH polarization images. The model is a binary classifier trained on the target classes, detected-ship and non-detected ship using SAR-based geophysical and geometrical features, i.e. wind speed, sea state and incidence angle, and Automatic Identification System (AIS)-based construction feature, i.e. ship length. Once the model is trained, the optimal hyperplane separating the two target classes, can be used to predict the probability of detection as function of the different input features. In particular in Figure 1 are shown the results for moderate sea state condition (significant wave height between $1 \mathrm{~m}$ and $4 \mathrm{~m}$ ) grouped according to ship length $L$ in 3 classes: small $(1<L[m] \leq 25)$, medium $(25<L[m] \leq 150)$ and large $(150<L[m])$. 


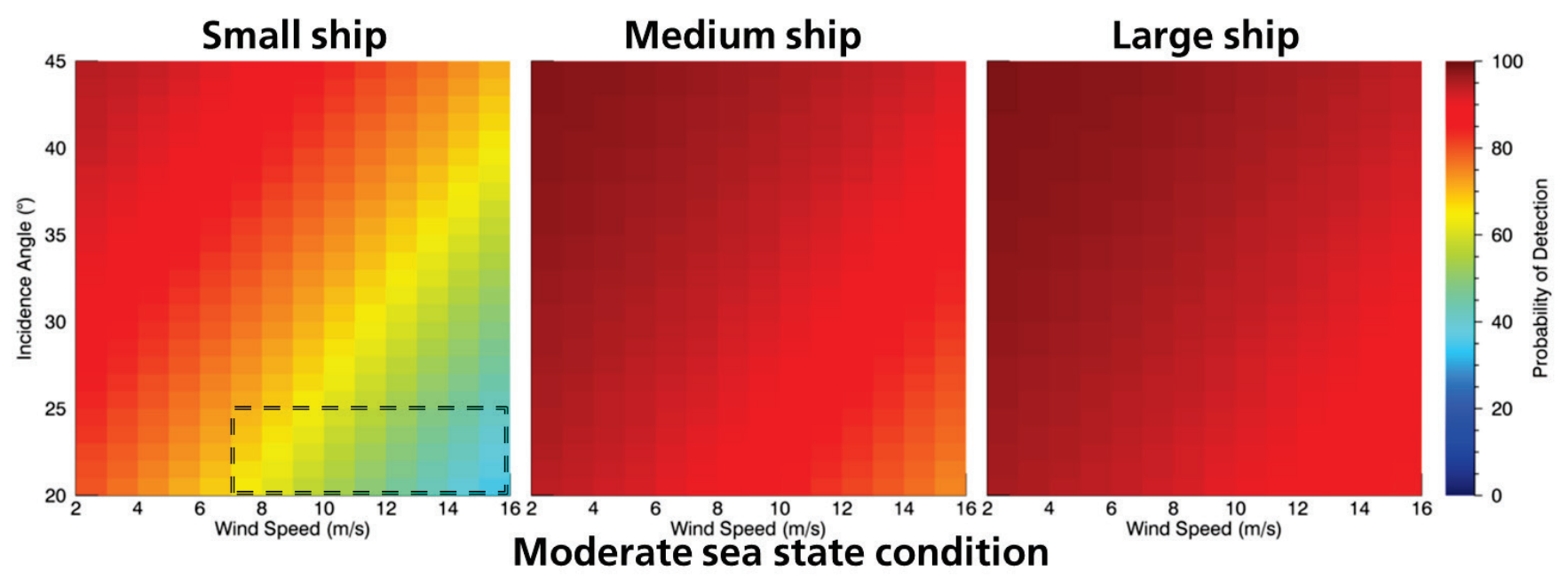

Figure 1. TerraSAR-X high resolution data-driven ship detectability model results for different ship sizes (from left to right: small, medium and large vessels) for the case of moderate sea state conditions. Each plot shows the probability of detection in function of incidence angle and wind speed.

\section{MOTIVATION}

As known, the capability of electronically steering the radar antenna beam in elevation reduces the maximum waiting time to cover any point on Earth by a remote sensing satellite. The new generation of SAR satellites, like TS-X, can exploit this feature to reduce the revisit time and increase the number of observations of a certain area of interest. This flexibility is particularly important in maritime surveillance and near real time (NRT) ship detection services. It is majorly significant that the detector is robust for the different viewing geometry and that the performances are somehow homogenous. As shown in the middle and right panel of Figure 1, exploiting the relatively high spatial resolution achievable with TS-X Stripmap mode, the detection of medium and large ships is almost a certainty for all conditions. A completely different story is for detecting small ship. In the left panel of Figure 1, a strong decrease in the probability of detection is visible going from the top left corner (preferable conditions) to the bottom right (worst condition, highlighted by the dashed rectangle). These results tell us that a ship detection algorithm based on radar backscatter with constant false alarm rate (CFAR) setting can achieve small ship detection performance close to $40-60 \%$ with a pre-selected probability of false alarm less than $10^{-13}$. Based on our experience, such a low probability of false alarm is necessary for operational SAR ship detection service at X-band.
In the literature several alternatives to the ship detection algorithm based on radar backscatter have been proposed exploiting amplitude and phase information of single look complex (SLC) SAR data. In particular [6]-[9] have shown that time-frequency technique, either performed in range or azimuth, perform better than single polarization intensity backscatter as ships tend to be coherent among the extracted looks while surrounding ocean de-correlate fast. To include the information provided by multipolarization channels, the polarimetric time-frequency correlation indicator has been introduced in [10] for full-polarimetric data and in [11] for dual-polarimetric data, showing very good results in the characterization of ships. However, in [12] Greidanus found that several known small fishing vessel of $30 \mathrm{~m}$ length were characterized by low cross-correlation between 3 looks generated from RADARSAT and ENVISAT image chip. Greidanus explained this outcome as due to the displacement of the ship signature peaks in different looks caused by waves induced ship motion.

In this analysis we use TS-X which is characterized by shorter integration time compared to RADARSAT and ENVISAT, therefore it is not expected the ship signatures behavior noted in [12]. This paper addresses the performance of timefrequency based detectors for the challenging conditions where small ships are imaged with large grazing angle and the surrounding met-ocean condition drastically reduce or vanish the ship-sea contrast. In this sense this work represents a novelty in this field compared with previous research papers as either a) 


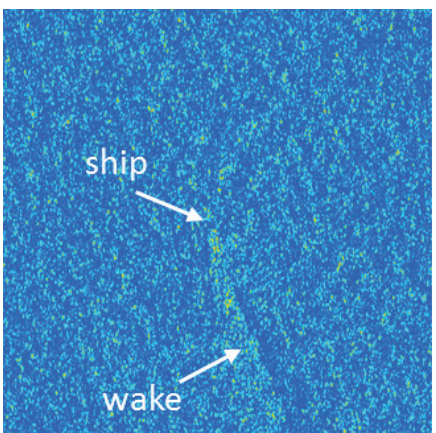

(a)

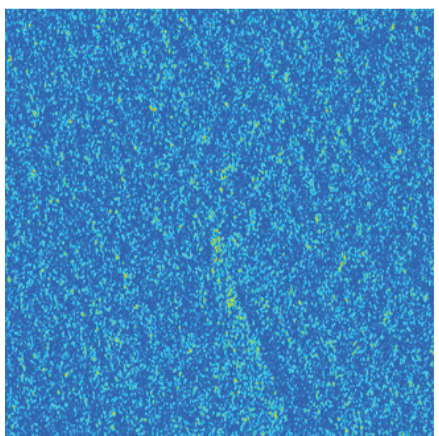

(b)

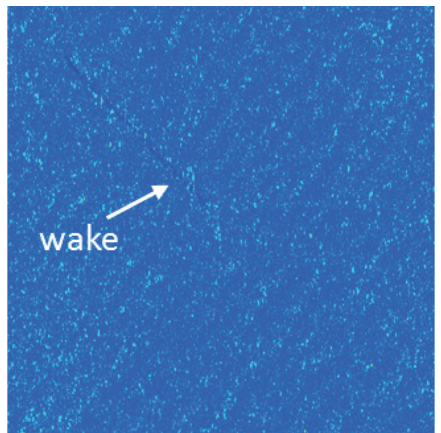

(c)

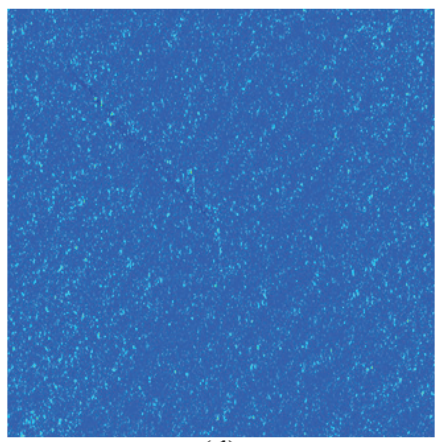

(d)

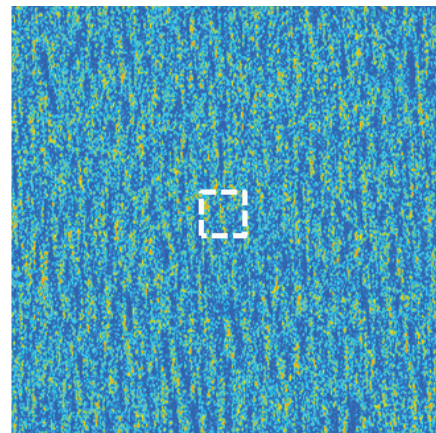

(e)

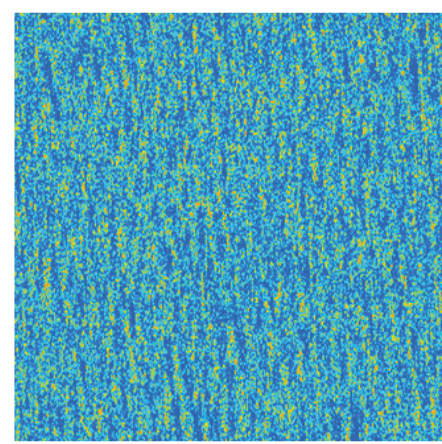

(f)

Figure 2. SAR patches containing small ships which have not been detected using intensity CFAR algorithm. Top row HH polarization; bottom row VV polarization. (a)-(b) sample where ship and wake are visually visible; (c)(d) only wake; (e)-(f) high clutter no ship or wake visible.

the number of ground truth small ship is limited as in [8], [10]-[12]; b) the conditions are general in terms of ship size, met-ocean condition and incidence angle as in [6], [7], [9]. The final aim is to assess this tool for TS-X data taking into account near real time (NRT) ship detection requirements.

\section{APPROACH AND DATA}

In order to validate and tune the in-house SAR ship detection algorithm, a large dataset composed of TS-X image products and ship's self-reporting messaging system, e.g. Automatic Identification System (AIS), has been collected. The SAR images included in the database provide an adequate number of samples for the different operational situations, e.g. data is collected using all combinations of available antenna beams from near range $\left(\sim 20^{\circ}\right)$ to far range $\left(\sim 45^{\circ}\right)$. SAR data duplication using repeat pass acquisitions has been necessary to capture the variability in the wind speed and sea state as well as in the presence of ship size samples. The complete SAR database has been processed with the in-house CFARbased SAR and AIS INtegration Toolbox (SAINT), which is also able to estimate the local wind speed and significant wave height using an appropriate geophysical model function (GMF).

As expected from the model prediction in Figure 1 , most of the missed detections (certified by the transmitted AIS messages) occur when the wind speed and sea state start to become moderate and the radar incidence angle is close to $20^{\circ}$. This sub-dataset represents the baseline for the performance analysis of this study. To evaluate simultaneously both co-pol channels on the same ship samples, the coherent dualpol HH-VV TS-X imaging mode has been used for the SAR data collection.

The time-frequency technique used in this study is a well know signal processing method which is based on following steps: 1) Fourier transformation of the data (in range, azimuth or both directions); 2) correct an eventual residual Doppler centroid shift; 3) spectrum whitening and sub-spectrum selection; 4) inverse Fourier transform. Further details can be found in [8]-[10]. A fixed number of 2 non overlapping subspectrums are used in this study.

\section{RESULTS}

Figure 2 illustrates three examples of non-detected small ship (from left to right, $36 \mathrm{~m}, 23 \mathrm{~m}$ and $17 \mathrm{~m}$ 

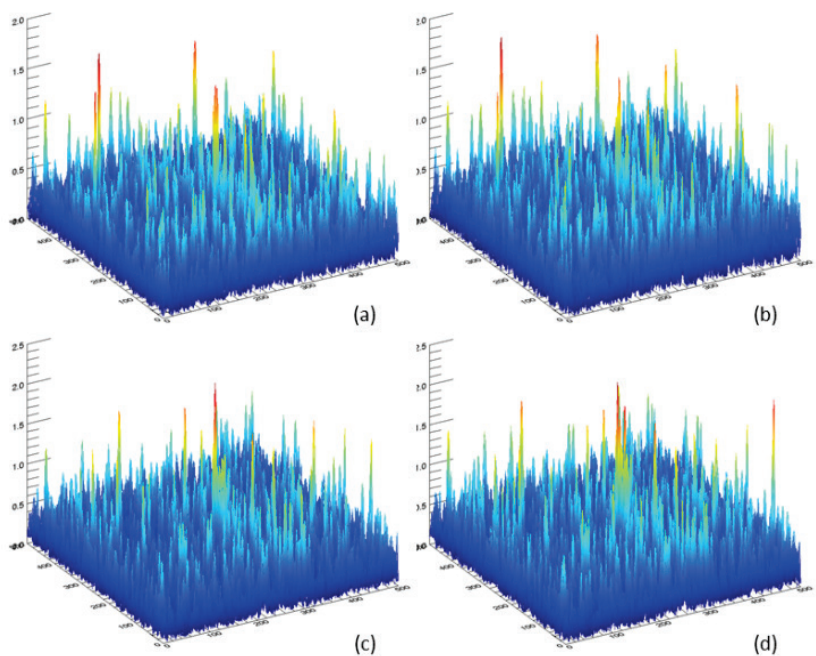

Figure 3. Surface plots of the cross-correlation results. (a)-(b) $\mathrm{HH}$ and $\mathrm{VV}$ after azimuth decomposition. (c)-(d) $\mathrm{HH}$ and $\mathrm{VV}$ after range decomposition.

length). The top and bottom rows show the intensity of the $\mathrm{HH}$ and VV channels, respectively. Due to the large grazing angle of observation and moderate metocean conditions (wind speed $>8 \mathrm{~m} / \mathrm{s}$ ) the ship-sea contrast is very limited (see (a)-(b)), vanish for the ship but not for the wake (see (c)-(d)) or is completely absent for both the moving ship and its wake (see (e)(f)). Please note that the presence of a ship in all samples included in our analysis is ensured by the geolocation information provided by AIS. Ship speed and course over ground (COG) information is here used to center a region of interest (ROI) of 501x501 pixels on the expected ship position imaged by SAR.

The cross-correlation of each co-pol channel is evaluated using a boxcar window of $5 \times 5$ pixels after time-frequency decomposition in range and azimuth. To save space, the surface plots of the crosscorrelations obtained using the $\mathrm{HH}$ and $\mathrm{VV}$ channel is shown only for the case of Figure 2 (a)-(b). Mean target to clutter ratio (TRC) measured with reference to Figure 2 (a)-(b) and Figure 3 (a)-(d): 1.72, 1.61, $1.46,1.27,1.73$ and 1.47. Similar trends are experienced for the other cases.

\section{REFERENCES}

[1] P. W. Vachon, J. W. M. Campbell, C. A. Bjerkelund, F. W. Dobson, and M. T. Rey, "Ship Detection by the RADARSAT SAR: Validation of Detection Model
Predictions," Can. J. Remote Sens., vol. 23, no. 1, pp. 48-59, Mar. 1997.

[2] P. W. Vachon, J. Wolfe, and H. Greidanus, "Analysis of Sentinel-1 marine applications potential," in 2012 IEEE International Geoscience and Remote Sensing Symposium, 2012, pp. 1734-1737.

[3] C. Bentes, D. Velotto, and S. Lehner, "Analysis of ship size detectability over different TerraSAR-X modes," in Geoscience and Remote Sensing Symposium (IGARSS), 2014 IEEE International, 2014, pp. 51375140.

[4] B. Tings, C. Bentes, D. Velotto, and S. Voinov, "Modelling Ship Detectability Depending On TerraSAR-X-derived Metocean Parameters," CEAS Space J., unpublished.

[5] M. G. V. Silva and H. Greidanus, "JRC-Frontex Spaceborne SAR Small Boat Detection Campaing Italy \& Spain," European Commission Joint Research Centre Institute for the Protection and Security of the Citizen, Luxembourg, JRC 67517.

[6] K. Ouchi, S. Tamaki, H. Yaguchi, and M. Iehara, "Ship detection based on coherence images derived from cross correlation of multilook SAR images," IEEE Geosci. Remote Sens. Lett., vol. 1, no. 3, pp. 184-187, Jul. 2004.

[7] J.-C. Souyris, C. Henry, and F. Adragna, "On the use of complex SAR image spectral analysis for target detection: assessment of polarimetry," IEEE Trans. Geosci. Remote Sens., vol. 41, no. 12, pp. 2725-2734, Dicembre 2003.

[8] C. Brekke, S. N. Anfinsen, and Y. Larsen, "Subband Extraction Strategies in Ship Detection With the Subaperture Cross-Correlation Magnitude," IEEE Geosci. Remote Sens. Lett., vol. 10, no. 4, pp. 786-790, Jul. 2013.

[9] A. Marino, M. J. Sanjuan-Ferrer, I. Hajnsek, and K. Ouchi, "Ship Detection with Spectral Analysis of Synthetic Aperture Radar: A Comparison of New and Well-Known Algorithms," Remote Sens., vol. 7, no. 5, pp. 5416-5439, Apr. 2015.

[10] C. Hu, L. Ferro-Famil, and G. Kuang, "Ship Discrimination Using Polarimetric SAR Data and Coherent Time-Frequency Analysis," Remote Sens., vol. 5, no. 12, pp. 6899-6920, Dec. 2013.

[11] F. Banda, L. Ferro-Famil, and S. Tebaldini, "Polarimetric time-frequency analysis of vessels in Spotlight SAR images," in Geoscience and Remote Sensing Symposium (IGARSS), 2014 IEEE International, 2014, pp. 1033-1036.

[12] H. Greidanus, "Sub-aperture Behavior of SAR Signatures of Ships," in IEEE International Conference on Geoscience and Remote Sensing Symposium, 2006. IGARSS 2006, 2006, pp. 3579-3582. 